\title{
URBANISATION AND 3D SPATIAL MODELLING- A GEOMETRIC APPROACH
}

\author{
E. E. Duncan ${ }^{\mathrm{a} * *}$ and A. Abdul Rahman ${ }^{\mathrm{a}}$
}

a3D GIS Research Laboratory, Faculty of Geoinformation and Real Estate, Universiti Teknologi Malaysia, 81310, Skudai, Johor Bahru, Malaysia - ededward2@live.utm.my / alias@utm.my

Commission II, WG II/2

KEY WORDS: Urbanisation, 3D Space, 3D TIN, 3D cadastre, 3D volume parcels

\begin{abstract}
:
Urbanisation creates immense competition for space, this may be attributed to an increase in population owing to domestic and external tourism. Most cities are constantly exploring all avenues in maximising its limited space. Hence, urban or city authorities need to plan, expand and use such three dimensional (3D) space above, on and below the city space. Thus, difficulties in property ownership and the geometric representation of the 3D city space is a major challenge. This research, investigates the concept of representing a geometric topological 3D spatial model capable of representing 3D volume parcels for man-made constructions above and below the 3D surface volume parcel. A review of spatial data models suggests that the 3D TIN (TEN) model is significant and can be used as a unified model. The concepts, logical and physical models of 3D TIN for 3D volumes using tetrahedrons as the base geometry is presented and implemented to show man-made constructions above and below the surface parcel within a user friendly graphical interface. Concepts for 3D topology and 3D analysis are discussed. Simulations of this model for 3D cadastre are implemented. This model can be adopted by most countries to enhance and streamline geometric 3D property ownership for urban centres. 3D TIN concept for spatial modelling can be adopted for the LA_Spatial part of the Land Administration Domain Model (LADM) (ISO/TC211, 2012), this satisfies the concept of 3D volumes.
\end{abstract}

Keywords: 3D TIN, Unified Spatial Model, Topology, Surface and Subsurface objects, Level of Details (LoD), Level of Realism (LoR).

\section{INTRODUCTION}

Urbanization is the concentration or migration of the inhabitants of a country to an area where socio-economic activities are high, such cities are usually planned with unique landmarks that make such places become tourist centres. Land in such areas is limited and the partition of such spaces has to be carried out above, on and below the city space. Spatial objects are features that can be located on the surface of the Earth, within a region and a country. These spatial objects can be described as two dimensional (2D) or three dimensional (3D) when the height (Z) value is added. Spatial representations of objects are unique, since ideally no coordinate pair can represent two different features on the surface of the Earth. 2D GIS is a knowledge based tool through topology which establishes knowledge between the various geometric primitives such as points (or nodes or vertices), lines (arcs or segments or edges) and polygons (areas or faces) together with their semantic properties. 2D GIS has several limitations such as the visualization of feature objects as points, lines and polygons, difficulty in interpreting some 2D maps due to hidden features, difficulty in visualizing utility networks due to inadequate information on height (Abdul Rahman et. al., 2012) and 2D map generalization. Hence, most 2D maps are inaccurate and can also not be relied on for $2 \mathrm{D}$ cadastre, this stalls developments in most city centres, where these 2D cadastre maps introduces a high level of complexities. The visualisation of feature objects within a digital environment representing reality is the basis of 3D GIS. With the introduction of height $(Z)$ above sea level, features can be realised in $3 \mathrm{D}$, users could also visualise $2.5 \mathrm{D}$ in relation to the Earth's surface, and this forms the digital terrain model (DTM). 3D data is obtainable from varied sources such as Computer Aided Design (CAD) drawings and can be represented in any 3D data model using the basic geometry element i.e. the node for the vector environment or orthophotos and orthoimagery from digital photogrammetry or remote sensing respectively, the basic geometry is the voxel for the raster environment.

Currently different spatial data models have been proposed by different researchers for 3D GIS, these spatial data models are different in presentation, usage and application. There is the need to collate all the existing spatial models and study the spatial data model that can best represent a unified spatial data model for all features above, on and below the surface. Researchers are required to start thinking of a unified spatial model that cuts across all possible applications with the following characteristics: the model should be easily understandable, easy to use and with proven sound mathematical background.

According to OGC (2012), the spatial model of city objects are based on ISO 19107 standard which represents 3D geometry according to the b-rep of Foley et. al., (1995). CityGML avoids the repetition of faces with topology being maintained, however the problem of leaving holes may persist and the concept of a composed object without holes necessary to achieve a model. CityGML has made topology optional but currently, a major issue in 3D GIS. In this research topological directional adjacency is considered to exploit the concept of above, beside

*ededward2@live.utm.my 
and below adjacency relations. CityGML supports the modelling of underground buildings and tunnels but the integration of the above, on and below objects has not been achieved. Standards such as CityGML, GeoSciML and Industry Foundation Classes (IFC) has been developed for 3D spatial artificial and natural features above and below the Earth's surface, these features have not been integrated due to challenges in geometric, topologic and semantic heterogeneities. However the integration of these standards is under consideration by OGC (2013).

\section{REVIEW OF SPATIAL DATA MODELS}

Previously, representing land parcels have not been a problem, since the land parcel and building footprints can be captured as a polygon. Currently, for city or urban space, 3D maps or 3D city models are becoming more important for city planners and the request for 3D spatial models for volume parcels have become a challenge. The benefits for 3D modelling are: accurate inventory, increase in revenue collection, effective planning schemes and accurate positioning of $3 \mathrm{D}$ objects above, on and below a land parcel. A review of current 3D spatial data models and a selection of one befitting a unified spatial data model for most applications selected that may also be the unique model for 3D GIS is indicated.

Surface 3D objects in the case of 3D city models have been studied by various researchers using the vector approach (Molenaar, 1990; Abdul Rahman, 2000; Zlatanova, 2000; Coors, 2003). 3D data models such as the tetrahedral network (TEN) (Pilouk, 1996) the constrained tetrahedral network (TEN) (Penninga and Oosterom, 2008), the Object Oriented Data Model (OODM) (Wang, 2006; Koshak and Flemming, 2002), Urban Data Model (UDM) (Coors, 2003) and the Simplicial Simplex Model (SSM) (Zlatanova, 2000) approaches have been used for 3D city modelling.

Surface and subsurface unified models have been studied by researchers: Abdul Rahman and Pilouk, 2008; Wang, 2006; Breunig and Zlatanova, 2006; Zhou et. al.,, 2008. The principle behind their research was enhanced forms of 3D FDS for 3D objects using 3D spatial data for objects above the surface and below the subsurface. The 3D FDS (Molenaar, 1990) was proposed for 2D GIS, but this has been adopted and made applicable by researchers for 3D GIS. The model has the point, line, surface and body as the entity object from the basic geometry of the node with topological relations of is in, is on, left and right.

Subsurface objects, in the areas of geology, hydrogeology, geotechnical, oceanography and geophysics have been studied extensively culminating in software's such as Leapfrog, Vulcan, Rockware and the GeoTool Kit amongst others. These deal with natural objects such as the spatial distribution of threedimensional (3D) continuous geological stratigraphy, borehole information and ore modelling of a mineral underground. These feature objects are referred to as fields whilst the object view approach considers the space as being 'empty and populated with discrete entities such as buildings, roads etc. (Ledoux and Gold, 2008). Researches for subsurface 3D models were aimed at modelling the natural subsurface: Abdul Rahman (2000) used the 3D TIN for drill hole locations. This idea was extended by Wang (2006) to include city modelling. Breunig (2001) carried out extensive research on geological bodies (GeoToolKit) using the TEN model. Zhou et. al.,, (2008) also used the cell complex and implemented the model for geology. The OO3D-ISDM spatial data model (Wang, 2006) was used for the integration of city objects and drill hole data based on the concept of 3D TIN. Wu and Shi, (2004) proposed a spatial data model based on GGTP and E-GTP the generalized tri-prism (GTP) for geosciences applications, these models uses the concept of 3D TIN in its implementations. The cell complex which is an enhanced concept from 3D TIN was applied as an integrated model in the area of city objects and geology (Pigot, 1992 and Zhou et. al.,, 2008). Researchers have enumerated the need to integrate manmade objects for both the surface and subsurface (Emgard and Zlatanova 2008; Zhou et. al.,, 2008; Ledoux and Gold, 2008; Zlatanova, 2008; Gold, 2008). Concepts from current data models (Abdul Rahman and Pilouk, 2008; Wang, 2006; Wu and Shi, 2004) which investigated models for above, on and below surface objects basically for geology, can be extended to include man-made objects for the subsurface. Most of the spatial models investigated showed the TIN and 3D TIN in a hybrid approach by integrating TIN with other 3D representations.

Abdul Rahman (2000) used the 3D TIN in a 2D and 3D TIN hybrid approach to represent 3D simple buildings with terrain and borehole representation for the subsurface. Pilouk (1996) used the 3D TEN (TIN) to represent geological features. Concepts for volume parcels for 3D cadastre have been presented by Abdul Rahman et. al., (2012) and Lemmen (2012) and the quest for a unified spatial data model still under research. This research further enhances these concepts and exploits the concept for 3D cadastre and implements these concepts with Level of Detail (LoD) concepts for texturing buildings in 3D cadastre. This research highlights the tetrahedron as basic geometry for 3D modelling for features above, on and below the earth surface, be it natural or artificial. The 3D TIN (TEN) model is presented as the unified spatial data model which when fully explored could be the panacea for realising 3D GIS. This research is to further contribute to this research goal.

\section{CONCEPTS}

The concept is based on tetrahedral meshes which can be degenerated to the cube as the basic 3D geometry such as the cube and other regular or irregular shapes. An enhanced 3D TIN model is proposed, with the solid being formed by tetrahedrons or polyhedrons. This presents a solid composed of tetrahedrons, surface composed of triangles, a line is composed of arcs or segments and point consists of nodes. The concept is shown in Figure 1.

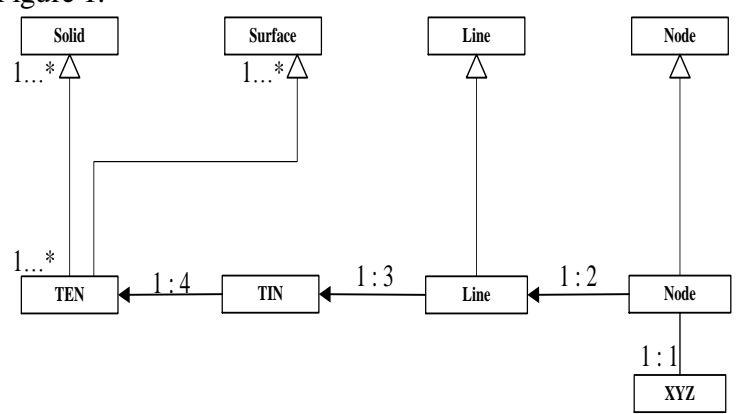

Figure 1. Concept for the 3D Unified Spatial Data Model

Nodes are formed by geographic coordinates XYZ which can form point features such as lamp posts, fire hydrant features and are part of the city furniture. Two nodes is part of the line or segment and can be expressed as a beginning node and an ending node. Three of such lines or segments will become part 
of the edge of the TIN surface. TIN surfaces will have edges or lines which will be shared and create the shared sub feature level (Pilouk, 1996) for a multi-theme model. The concept of implicit geometry and the merits of this geometry is well explained by OGC (2012) is used to form the nodes with appropriate identifiers, the line identifier will have a beginning node and an ending node with the topological relationship of is on, is in, point and solid, line and solid, line and surface, point and surface being used to form the relationship for the unified model. The 3D TIN is formed by four nodes, six lines and four triangles, to form solids for both the surface and subsurface. The model is being adopted due to the fact that it can be used to model regular and irregular objects and can thus represent features above, on and below the earth surface. Using the cube as the basic feature for volume objects, the cube can be decomposed to five or six tetrahedrons at the geometry level. The 3D TIN model is an improvement over the 3D FDS model, this consists of the tetrahedron representing the body object, triangles representing the surface, lines as arc or edge objects and the node as point objects representing geometric (simplex), sub feature (simplex complex) and complexes. The topological relationship is given by the adjacency of spatial objects.

This research focussed on vector based data to achieve its goals: to represent a unified spatial data model capable of being used as a generalized spatial data model for 3D GIS. The logical model is presented in Figure 2.

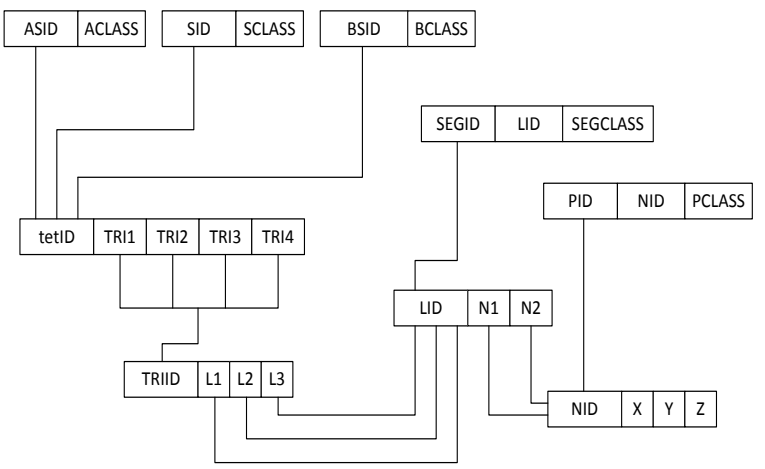

Figure 2. The Logical model

The classes for the various entities or features will then be connected or joined to the attributes to complete the database structure of the model. The above class ACLASS and surface class SCLASS and the below class BCLASS are all connected to the tetrahedron tetID to form the unified spatial data model, topology maintained between the faces. The significance of this is that tetrahedrons can be used to represent all features above, on and below the Earth's surface.

The concept for a unified object based on man-made features for above, on and below the earth surface is presented in Figure 3. This shows man-made features connected to the terrain model for above and below surface features and man-made features which are independent of the above and below features. Features for the latter have to be topologically connected in order to show above surface features, which have independent features below the surface.

Emgard and Zlatanova (2008) presented conceptual models for an integrated model within CityGML, and presented the terrain intersection curve (TIC) within that framework. This paper is advocating for a stand-alone platform which uses the 3D TIN with only solid tetrahedrons being formed for the integration of the above, on and below 3D spatial objects.

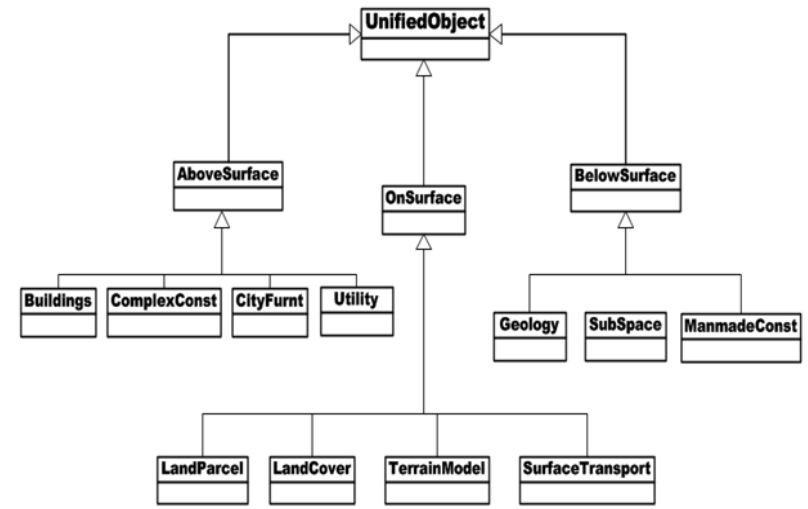

Figure 3 UML Concept for UnifiedObject

This paper is advocating for a stand-alone platform which uses the 3D TIN as a unified spatial data model for the integration of the above, on and below 3D spatial objects. This concept will also attract applications such as the network of horizontal and vertical cavities in the case of $3 \mathrm{D}$ mining. $3 \mathrm{D}$ city objects have underground facilities such as storage, tunnels, cavities, underground rail systems and car parks, knowledge about these man-made objects are required for 3D cadastre.

From the concepts presented, the requirements for the unified spatial data model: are to construct a 3D geometry capable of representing regular objects (such as buildings) and irregular objects (geological body, a spaghetti tunnel or transportation network or mine underground workings); the unified spatial data model should have sound or established mathematical background (counter clockwise algebraic topology in relation to simplexes or clockwise algebraic topology in relation to complexes); support for topology, this must indicate the relationships between geometric objects, consistency constraints must be indicated and directional adjacencies such as beside, above and below highlighted.

\section{3D TOPOLOGY CONCEPTS FOR THE UNIFIED MODEL}

2D GIS is well expressed and clear on topology as it clearly demonstrates the properties of, connection, adjacency and containment. This expresses knowledge using the geometric properties of node, arc or line or edge and area or polygon. Research on 3D topology is still in the implementation stage as the topology between volumetric geometries is still not clear. Spatial database management systems such as Oracle Spatial $11 \mathrm{~g}$, PostgreGIS and Bentley have been used to implement some topology for specific applications. The spatial data model with its corresponding data structure is the core for 3D GIS software development. 3D topology model is in analysing 3D spatial phenomenon in the real world due to the spatial relations between each object. However the topology of 3D spatial objects is more difficult to analyse than $2 \mathrm{D}$ topology due to the complexity of $3 \mathrm{D}$ geometry. Therefore the implementation of topological operators in 3D spatial DBMS is more difficult than spatial DBMS for 2D spatial objects due to geometric complexity (Kim et. al., 2008).

Current researches focus on the implementation model and storage using 3D geometry in terms of 3D topological relations i.e. disjoint, meet, Equal, inside, contains, covers, coverby and overlap (Egenhofer and Sharma 1993, Chen et. al., 2008) for 3D spatial analysis. 3D topological operations cover the relationship of 4-intersection model ( 8 kinds of relations), this could be 
extended to 9-intersection model. A B-rep model defines spatial objects by their bounding elements e.g. planar faces. Nodes and edges are defined by intersection of the bounding planes. B-rep model has been described as the model which constitutes the most popular model to store 3D topological data (Choi and Lee, 2009). 3D GIS analysis could also be implemented for 3D data type and functions. Although the theoretical number of the 9intersection model is $2^{9}$ i.e. 512 relationships, only a small number of these can be seen in reality (Zlatanova 2000, Kurata 2010).

The topological structure for the unified model uses the basic solid geometry for 3D TIN which is the tetrahedron and further enhanced by incorporating some volume and quality statistics within a graphical user interface. This concept is adopted for the following reasons: It is possible to represent regular and irregular objects, the model is mathematically sound with topology being established geometrically by avoiding repetition of data, the condition of no gaps or overlaps are satisfied.

Spatial objects can be represented by boundary elements such as vertices, edges and face, with topology being introduced by the concept of adjacency. The concept of directional adjacency is employed to depict topological relations above, beside and below for the unified spatial data model. The Boundary representation forms objects such as edge, face and volume using the node as the geometric primitive. The 3D TIN is significant and has been explored in a hybridised form in most spatial data models. An attempt is made to represent the 3D TIN using the tetrahedron concept as a unified spatial data model for 3D modelling for all objects above, on and below the city space, data for face sharing are not repeated.

\section{IMPLEMENTATION}

This research implements the unified spatial data model using Microsoft Visual C++ version 2008 programming software was used in conjunction with OpenGL and Qt libraries to create a prototype for the unified model. Implicit data using the Boundary representation (B-rep) was used to create a tetrahedral wireframe, which is then degenerated to form the basic volume, within a graphical user interface (GUI) capable of displaying the data structure and the attributes. The integrity of the tetrahedrons formed are checked using a quality check, the volume and centre of mass of the basic volume spaces determined, statistical inferences in relation to the quality of the tetrahedron are then computed.

Implicit geometry is used to form the nodes with appropriate identifiers, the line identifier will have a beginning node and an ending node with the topological relationship of is on, is in, point and solid, line and solid, line and surface, point and surface being used to form the relationship for the unified model. The tetrahedron is formed by four nodes, six lines and four triangles, to form solids for both the surface and subsurface. Six regular tetrahedrons form the basic cube which is the basic volume primitive in 3D GIS

The merit of such an algorithm is that no special equipment is required as these data sources already exist in the form of AutoCAD data, orthophotos, orthoimagery, GPS data, mine data and data from Light Detection and Ranging (LIDAR) equipment. These data can then be represented as explicit or implicit geometry (OGC, 2012).
Figure 4 is the tetrahedral mesh generated for the basic cube. The basic geometry cube created from the tetrahedron with the volume and centre of mass computed, the volume is significant as it shows the 3D space occupied and the centre of mass of the cube used to enhance navigation within the block or described the block in an attribute database. Figure 5 is the concept of 3D volume spaces degenerated form a tetrahedral mesh.

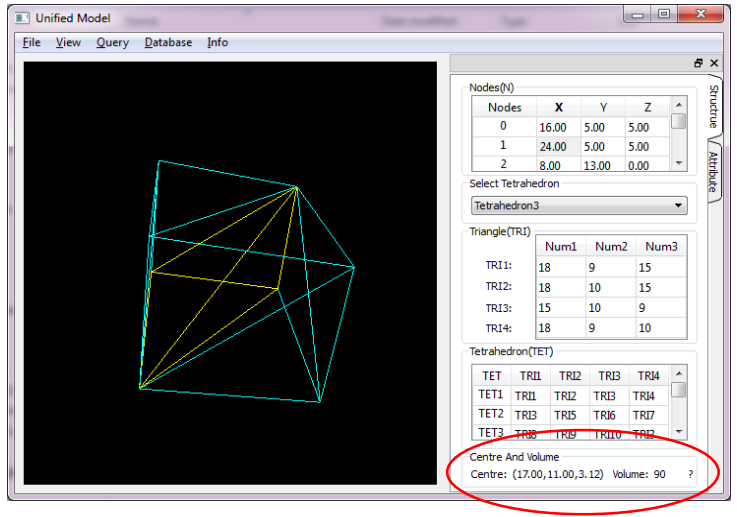

Figure 4. Cube generated from tetrahedrons for volume parcels

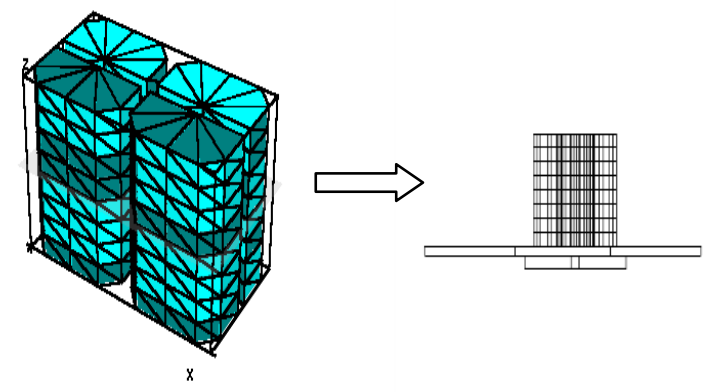

Figure 5. Tetrahedral mesh degenerated to 3D volume spaces

Figure 6 is the graphics user interface (GUI) showing the wireframe and additional polygons generated for texturing. In this research, indoor navigation has not been explored.

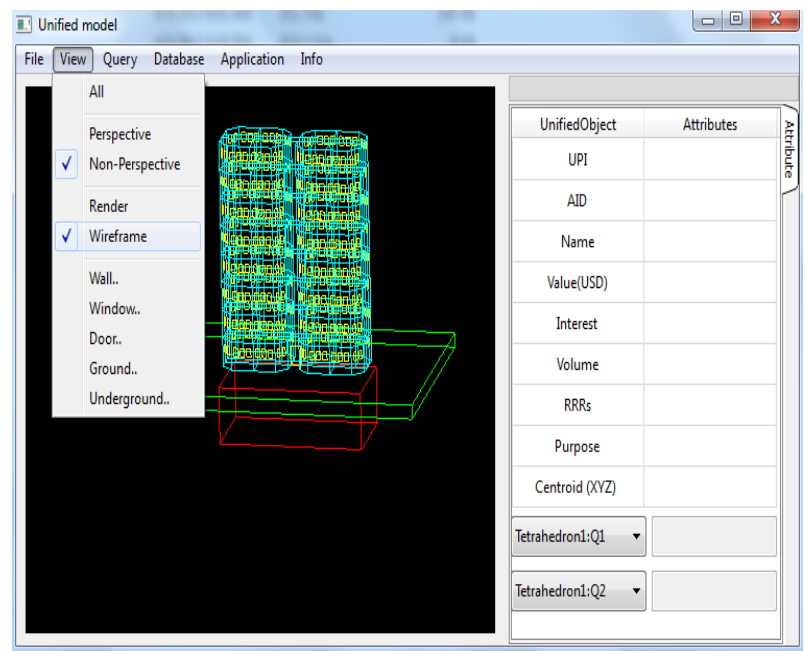

Figure 6. Tetrahedral mesh for a building block degenerated to simplified unified block. 
Figure 7 shows a three storey building with subsurface manmade construction for parking. The graphical part is on the left with the semantic information on the right of the GUI showing a queried part. This concept can be extended for any multilevels.

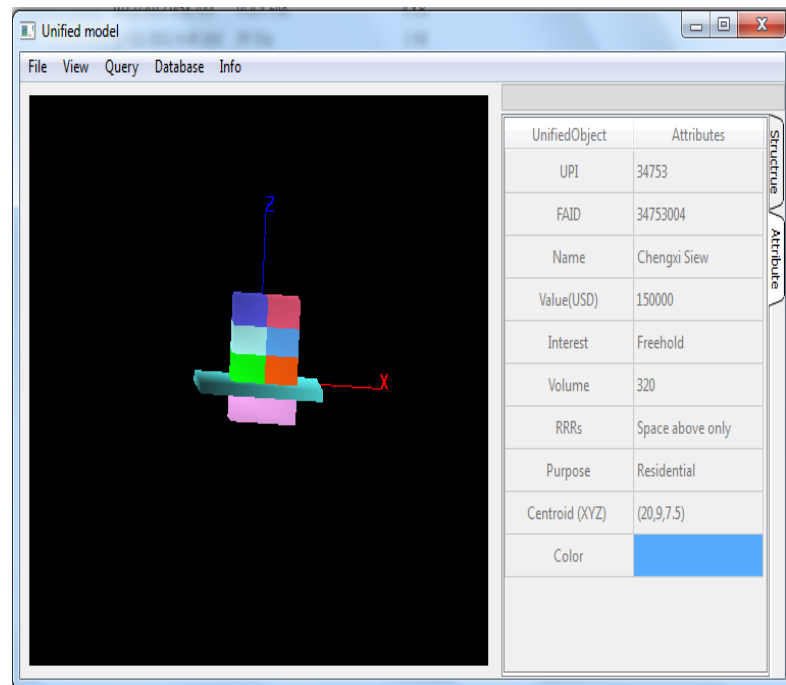

Figure 7. Application for 3D cadastre.

Texturing is the technique of increasing the Level of Realism (LoR) of 3D objects which among others include pictometry in the capture of appropriate images for the various components of a building such as window, door, and roof types to increase LoR. In this research, texturing using a hybridised rendering tool was used. This uses the colour code Red, Green and Blue (RGB) and images of windows and doors to fit a specific window and door size. The significance of this part of the research was to give cadastral blocks some level of realism. This is a complex cadastral problem as it raises questions such as who owns the flats above the road. Who owns the land parcel under the road? What are the tax liabilities of these owners? What is the size of cavities or tunnels created or occupied beneath the surface? What are their liabilities? The unified spatial data model can provide answers to such queries. Figure 8 shows a textured block for 3D cadastre.

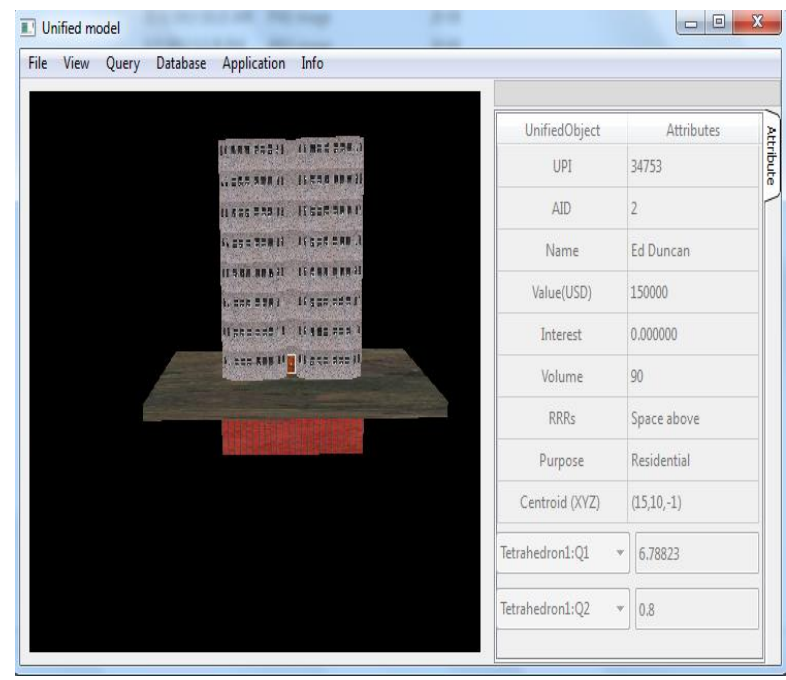

Figure 8. A textured unified block for 3D Cadastre

\section{CONCLUDING REMARKS}

The 3D TIN is significant and satisfies the requirement for a unified spatial data model. The 3D TIN model can represent features that are regular and irregular, artificial and natural. The merits of the unified spatial data model has been enumerated. The concept for a new unified model (3D TIN) can integrate above surface objects and below surface geology and man-made objects. The conceptual model for the unified model is presented and directions for topology and analysis indicated. An algorithm to fully integrate the above framework in a database has been enumerated. Topological inferences to query above, beside and below objects has been carried out for 3D buildings, this is significant for 3D cadastre in an urban centre. The GUI for 3D TIN will be enhanced to show more derived statistical information in the next implementations. The concept for 3D volume parcels using 3D TIN is valid and can find applications for 3D cadastre for urban or city centres to bring about sustainable development. This research discusses possibility of using the tetrahedron for 3D modelling. This has been implemented for 3D cadastre and the possibility of realising 3D GIS highlighted as the author's contribution.

If 3D GIS application software is to be realised then the concept of a unified spatial data model is to be adopted and enhanced further. The applications enumerated are representations of complex situations in the real world and shows some of the complexities that can be encountered. The next phase for this research will focus on an outdoor and indoor application for 3D cadastre and facility management.

Acknowledgements: We would like to convey our deepest acknowledgement, to the 3D GIS Research Lab of the Faculty of Geoinformation and Real Estate, University of Technology Malaysia, Malaysia for providing the enabling environment for research. Our appreciation also goes to the University of Mines and Technology (UMaT) of Ghana for sponsoring the researcher.

\section{References}

Abdul Rahman, A., Van Oosterom, P. Hua, T. C. Sharkawi, K. H., Duncan, E. E., Azri, N. and Hassan, I. H. (2012). 3D Modelling for Multipurpose Cadastre 3rd International Workshop on 3D Cadastres: Developments and Practices 25-26 October 2012, Shenzhen, China, pp 185 -202.

Abdul-Rahman, A., (2000). The Design and Implementation of a Two and Three dimensional Triangular Network based GIS, PhD Thesis, University of Glasgow, U. K., pp. 204.

Abdul-Rahman, A. and Pilouk, M., (2008). Spatial Data Modelling for $3 D$ GIS, published by Springer BerlinHeidelberg, New York, pp. 289.

Breunig, M., Cremers, A., Muller, W. and Siebeck, J. (2001). New Methods for Topological Clustering and Spatial Access in Object-Oriented 3d Databases, $9^{\text {th }}$ ACM International Symposium on Advances in Geographic Information Systems.

Breunig, M. and Zlatanova, S., (2006). 3D Geo-DBMS. Zlatanova, S. And Prosperi, D. (eds.), Large-scale 3D Data Integration-Challenges and Opportunities: 87-115. London: Taylor and Francis. 
Chen, T.K., Abdul-Rahman, A., and Zlatanov, S., (2008). 3D spatial operations for geo-DBMS: geometry vs. topology. The International Archives of the Photogrammetry, Remote Sensing and Spatial Information Sciences. XXXVII, part B2, pp. 549-554.

Choi, J. and Lee, J. (2009). 3D Geo-network for Agent-based Building Evacuation Simulatio, 3D Geo-Information Sciences, Lecture Notes in Geoinformation and cartography, 2009, Part II, pp. 283-299.

Coors, V., (2003). 3D-GIS in Networking Environments, Computer, Environments and Urban Systems, Vol. 27/4, Special Issue 3D Cadastre, pp 345 - 357.

Egenhofer, M. and Sharma, J. (1993). Assessing the consistency of complete and incomplete topological information. Geographical Systems1 (1): 47-68.

Emgard, L. and Zlatanova, S., (2008). Design Of An Integrated 3D Information Model, in Coors, V., Rumor, A., Fendel, E., and Zlatanova, S., (eds.) Urban and Regional data Management, UDMS Annual 2008, Taylor and Francis Group, London, pp. 143-156.

Foley, J., van Dam, A,. Feiner, S., and Hughes, J., (1995). Computer Graphics: Principles and Practice, Addison Wesley, 2nd Ed.

Gold, C., (2008). Chapter 25 Working Group III-ModellingPosition paper on Modelling 3D Geo-Information. In Advances in 3D Geoinformation Systems, van Oosterom, P., Zlatanova, S., Penninga, F., Fendel, E., (eds). published by Springer Berlin-Heidelberg, New York, pp. 429-435.

Kim, H., P. Waddell, V.N. Shankar, and G. F. Ulfarsson (2008). Modeling Micro-spatial Employment Location Patterns: a comparison of count and choice approaches. Geographical Analysis, 40(2), 123-151.

Koshak, N. and Flemming, U., (2002). Object Oriented Data Modelling and Warehousing to Support Urban Design, $6^{\text {th }}$ International Conference and Decision Support Systems in Architecture and Planning, July 7 - 10, 2002. Ellecom, The Netherlands.

Kurata, Y. (2010). An Overview of the Research on Topological Relations and Future issues in GIScience theory and Applications of GIS, 18(2), 41-51.

Ledoux, H. and Gold, C. M., (2008). Modelling Threedimensional Geoscientific Fields with Voronoi Diagram and its Dual, Int. J. of Geographical Information Science. Vol. 22. Nos 4 - 5, April - May 2008, 547 - 574.

Lemmen, C. (2012). A Domain Model for Land Adminstration, $\mathrm{PhD}$ Thesis, ITC dissertation number 210, ISBN 978906132 3365, 234pp.

Molenaar, M., (1990), “A Formal Data Structure for 3D Vector Maps", Proceedings of EGIS'90 Vol.2, Amsterdam, The Netherlands, pp. $770-781$.

OGC, (2012). Open Geospatial Consortium, CityGML Encoding Standard Version 2.0, OGC Doc No 12=019, 04-04-2012, Eds: Gerhard Gröger, Thomas H. Kolbe, Claus Nagel, KarlHeinz Häfele, 326pp.

OGC, (2013). Open Geospatial Consortium, the OGC and Geosciml4.0 Standards Working Group, http://www.opengeospatial.org/pressroom/pressreleases/179 4, (12 Mar 2013).

Penninga, F. and van Oosterom, P. J. M. (2008). A Simplicial Complex-Based DBMS Approach in 3D Topographic data Modelling, International Journal of Geographic Information Sciences, June-July, Volume 22, pp. 751 - 779.

Pigot, S., (1992). A Topological Model for a 3-D Spatial Information Systems, Proc. $10^{\text {th }}$ International Symposium on Computer Assisted Cartography (AUTOCARTO 10), Technical Papers, ACSM-ASPRS, Annual Convention, Vol. 6, Baltimore, Maryland, USA, pp 369-391.

Pilouk, M., (1996). Integrated Modelling for 3D GIS. PhD dissertation, ITC Publications No 40, The Netherlands, pp. 200.

Raper, J., (ed.) 1989. Three Dimensional Applications In Geographic Information Systems. Taylor and Francis Ltd., London, pp. 189.

Shi,W., Yang, B. and Li, Q. (2003). An Object-oriented Data Model for Complex Objects in Three Dimensional GIS, International Journal of Geographic information science (Quarterly), July-August, 7(5), pp. 411-430.

Shi,W. Z. (1996). A Hybrid Model for 3D GIS, Geoinformatica, Volume 1, pp. 400-409.

Wang, Y., (2006). 3D GIS Spatial Modelling for City Surface and Subsurface Integration, published by IEEE, pp. 1511 1518.

Wu, L and Shi, W., (2004). GTP-Based Integral Real 3D Spatial Model for Engineering Excavation GIS (E ${ }^{2}$ GIS), Geospatial Information Science (Quarterly) 7(3), pp. 123 128.

Wu, L. X., Shi, W. Z. and Christopher, G. M., (2003). Spatial Modelling Technologies for 3D GIS and 3D GMS. Geography and Geo-information Sciences 19 1, pp. 5-11.

Zhou, I., Lu, G., Sheng, Y., Xu. H., and Wang, H., (2008). A 3d GIS's Spatial Data Model Based on Cell Complex. The International Archives of the Photogrammetry, Remote Sensing and Spatial Information Sciences. Vol. XXXVII Part B2, Beijing, pp. $905-908$.

Zlatanova, S., (2000). 3D GIS for Urban Development, $\mathrm{PhD}$ Thesis, ITC Dissertation Series No 69. The International Institute for Aerospace Survey and Earth Sciences, The Netherlands.

Zlatanova, S., (2008). Acquisition-Position Paper-Data Collection and $3 D$ reconstructionand, Advances in $3 D$ Geoinformation Systems, van Oosterom, P., Zlatanova, S., Penninga, F., Fendel, E., (eds). published by Springer Berlin-Heidelberg, New York, pp. 425-428. 